\title{
XX. Long-distance telephony
}

\section{Prof. Perry F.R.S. \& H. A. Beeston}

To cite this article: Prof. Perry F.R.S. \& H. A. Beeston (1893) XX. Long-distance telephony , Philosophical Magazine Series 5, 36:219, 222-229, DOI: 10.1080/14786449308620468

To link to this article: http://dx.doi.org/10.1080/14786449308620468

$$
\text { 曲 Published online: } 08 \text { May } 2009 .
$$

Submit your article to this journal

LII Article views: 4

Q View related articles ¿ 
the ring along its core filament is allowable; but, as we have seen, this requires that the ring must be an extremely thin one, and in most cases of the coiling of wires for the conveyance of currents the assumption would be inadmissible; for, the ideal vortex filament required by the assumption (infinitely thin as compared with the radius of its aperture) finds but a very coarse representative in any coil of wire. We have already given the values of the magnetic force at the centre, $O$, of the curve formed by the wire and at the points, E, B, just outside its surface ; these are the analogues of the velocity of the (irrotationally moving) fluid at these points in the case of a vortex ring. The velocity with which the ring itself moves forward is given by Mr. Basset as equal to $\frac{f}{2 \pi a}\left(\log \frac{8 a}{c}-1\right)$, where $f$ is the strength of the vortex (product of the cross-section and molecular rotation), while the velocity of the fluid at $O$ is $\frac{f}{a}$; so that the ratio of the forward velocity of the ring itself to the velocity of the fluid at the centre, $\mathrm{O}$, of its aperture is

$$
\frac{\log \frac{8 a}{c}-1}{2 \pi},
$$

which, as Mr. Basset says, is "large" in the case supposed (i.e., a very thin ring). We must observe, however, when comparing actual electrical coils with fine vortex rings, that for a vortex ring for which $a$ is 1000 times $c$ (which would ordinarily be considered as a "fine" ring), this ratio is not very large : it amounts only to $1 \cdot 27$; while for a vortex ring the radius of whose aperture is 100 times that of its crosssection, this ratio is only 9 , i.e., the ring moves more slowly than the fluid at its centre.

XX. Long-Distance Telephony. By Prof. PkRRx, F.R.S., assisted by H. A. BEEsTON*

W HEN resistance, capacity, self-induction, and leakage are taken into account, this subject is one of considerable difficulty. It is given to very few men to be able to discuss complicated mathematical formula without making mistakes- - the proceedings of Scientific Societies possess many such mistakes detected and undetected-and consequently I instruct my students to experiment with their formulæ, using numerical values for their variables. The consideration of

$$
\text { * Communicated by the Author. }
$$


the most general problem of long-distance telephony, involving certain terminal conditions, has been taken up by Mr. Heaviside ; but the ordinary mathematical physicist must find great difficulty in understanding the investigation (Phil. Mag. January 1887). Some of my students have recently obtained numerical results, neglecting the terminal conditions, which seem to me to be very instructive, and I think that the Tables will have a permanent value.

As a matter of fact, the line is supposed to be of infinite length, and we consider the state of a signal as it gets further and further away from the origin.

By comparing the current $c$ at a section $x$ centimetres from the origin with the current at $x+d x$, and properly disposing of the difference, we arrive at the equation :-

$$
\frac{d^{2} c}{d x^{2}}=k l \frac{d^{2} c}{d t^{2}}+(k r+s l) \frac{d c}{d t}+s r c, . \quad . \quad .
$$

where (per unit length of conductor) $k$ is the capacity, $r$ the resistance, $l$ the self-induction, and $s$ the leakage conductivity. The solution which suits telephonic conditions is

where

$$
c=a \epsilon^{-h x} \sin (p t-g x),
$$

$$
\sqrt{\frac{k p r}{2}} \sqrt{\sqrt{\left(1+\frac{p^{2} l^{2}}{r^{2}}\right)\left(1+\frac{s^{2}}{k^{2} p^{2}}\right)} \mp\left(\frac{p l}{r}-\frac{s}{p k}\right)}
$$

gives the value of $l$ if the minus sign is taken, and gives the value of $g$ if the plus sign is taken, and $c=a \sin p t$ is the current at the origin. Of course $p=2 \pi f$, where $f$ is the frequency. Any number of such functions of any frequencies may exist simultaneously.

Two conditions must be satisfied in telephony. Taking the shrillest and gravest notes of the human voice as being of frequencies $f$ and $f^{\prime}$, and taking therefore currents of these frequencies :- let $\mathrm{X}$ be the distance at which the ratio of the amplitudes of the shrill and grave currents is increased by $1 / m$ th of itself; let $Y$ be the distance at which one of the currents has altered in lag behind the other by $1 / n$th of the periodic time of the more rapid one; then it is easy to see that

$$
\begin{aligned}
& \mathrm{X}=1 /\left\{m\left(h-h^{\prime}\right)\right\}^{*}, \\
& \mathrm{Y}=2 \pi /\left\{n p\left(\frac{g}{p}-\frac{g^{\prime}}{p^{\prime}}\right)\right\} .
\end{aligned}
$$

* This is approximate. If $m$ is not large, the true expression ought to be used,

$$
\mathrm{X}=\log _{\epsilon}\left(1+\frac{1}{m}\right) /\left(h^{\prime}-h\right) \text {. }
$$


The letters with dashes indicate that $p^{\prime}$ or $2 \pi f^{\prime}$ must be taken instead of $p$. It is easy to see that $\mathrm{X}$ and $\mathrm{Y}$ become infinite if $\frac{l}{r}=\frac{k}{s}$.

I do not know what values of $m$ and $n$ would produce confusion of sound in the telephone. But as an exercise we have taken $m=4$ and $n=6$. We have also taken $p=6000$, $p^{\prime}=600$.

For the first French Atlantic Cable the capacity and resistance were 0.43 microfarad and $2.93 \mathrm{ohms}$ per nautical mile, so that

$$
\begin{aligned}
& k=2 \cdot 3215 \times 10^{-12} \text { farads per centim. } \\
& r=1.582 \times 10^{-5} \text { ohms per centim. }
\end{aligned}
$$

Mr. Beeston has calculated the distances $\mathrm{X}$ and $\mathrm{Y}$, the lesser of which may be taken as the limiting distance for good telephony for various values of $l$ and $s$.

TABLE I.-Limiting distances $X$ in millions of centimetres for various amounts of leakage and self-induction. (One

\begin{tabular}{|c|c|c|c|c|c|c|c|}
\hline \multirow[b]{2}{*}{$\begin{array}{l}\text { Values of } \\
s \times 10^{10}\end{array}$} & \multicolumn{7}{|c|}{ Values of $l \times 10^{10}$} \\
\hline & 0 & $2 \cdot 6373$ & $26 \cdot 373$ & $79 \cdot 118$ & $131 \cdot 863$ & $184: 61$ & $263 \cdot 73$ \\
\hline 0 & 0.983 & 1.054 & 1.963 & $5 \cdot 169$ & $10 \cdot 178$ & $17 \cdot 500$ & 33.839 \\
\hline .01 & $\ldots$ & $\ldots$ & $1 \cdot 961$ & & & & \\
\hline$\cdot 10$ & ... & $\ldots$ & 1.969 & & & & \\
\hline 1 & $\cdots$ & $\cdots$ & 1.999 & & & & \\
\hline 5 & 1.049 & $1 \cdot 130$ & $2 \cdot 187$ & $6 \cdot 339$ & 13.944 & 41.532 & $75 \cdot 59$ \\
\hline 10 & $1 \cdot 130$ & $1 \cdot 224$ & $2-356$ & $8 \cdot 462$ & 21.98 & $59 \cdot 03$ & $328 \cdot 2$ \\
\hline 20 & $1 \cdot 319$ & $1 \cdot 444$ & $3: 357$ & $18 \cdot 009$ & $128 \cdot 17$ & $\begin{array}{l}\text { very } \\
\text { large }\end{array}$ & $131 \cdot 65$ \\
\hline 40 & $1 \cdot 754$ & 1.965 & 6.250 & $390 \cdot 11$ & 67.95 & 18.94 & $8 \cdot 75$ \\
\hline 70 & $2 \cdot 527$ & $2 \cdot 935$ & $17 \cdot 671$ & $39 \cdot 73$ & $7 \cdot 727$ & $4 \cdot 161$ & $2 \cdot 573$ \\
\hline 100 & $3 \cdot 447$ & $4 \cdot 134$ & $72 \cdot 03$ & $10 \cdot 00$ & 0.650 & $2 \cdot 111$ & $1 \cdot 617$ \\
\hline 150 & $5 \cdot 314$ & & & & & & \\
\hline 200 & $7 \cdot 536$ & & & & & & \\
\hline 250 & $10 \cdot 090$ & & & & & & \\
\hline
\end{tabular}
million centimetres are equivalent to about six miles.) 
TABLE II.-Limiting distances $\mathrm{Y}$ in millions of centimetres for various amounts of leakage and self-induction.

\begin{tabular}{|c|c|c|c|c|c|c|c|}
\hline \multirow[b]{2}{*}{$\begin{array}{l}\text { Values of } \\
s \times 10^{10}\end{array}$} & \multicolumn{7}{|c|}{ Values of $l \times 10^{10}$} \\
\hline & 0 & $2 \cdot 6373$ & $26 \cdot 373$ & $79 \cdot 118$ & $131 \cdot 863$ & $184 \cdot 61$ & 26373 \\
\hline 0 & $1 \cdot 459$ & $1 \cdot 484$ & $1 \cdot 778$ & $2 \cdot 665$ & 3719 & $4 \cdot 996$ & $7 \cdot 239$ \\
\hline .01 & $\ldots$ & $\ldots$ & $1 \cdot 783$ & & & & \\
\hline$\cdot 10$ & $\ldots$ & $\ldots$ & 1.800 & & & & \\
\hline 1 & $\ldots$ & $\ldots$ & 1.893 & & & & \\
\hline 5 & $1 \cdot 889$ & 1.927 & $2 \cdot 424$ & $4 \cdot 142$ & 6674 & $10 \cdot 472$ & 20.29 \\
\hline 10 & 2426 & $2 \cdot 482$ & $3 \cdot 297$ & 6.639 & $14 \cdot 130$ & 28.88 & $116 \cdot 36$ \\
\hline 20 & 3.752 & $3 \cdot 874$ & $5 \cdot 796$ & $18 \cdot 700$ & $82 \cdot 59$ & $\begin{array}{l}\text { very } \\
\text { large }\end{array}$ & $75 \cdot 12$ \\
\hline 40 & $7 \cdot 292$ & $7 \cdot 649$ & $15 \cdot 152$ & 56636 & $76 \cdot 55$ & $18 \cdot 184$ & $7 \cdot 214$ \\
\hline 70 & $15 \cdot 216$ & $17 \cdot 342$ & 57.965 & $76 \cdot 304$ & $11 \cdot 493$ & $5 \cdot 266$ & $2 \cdot 815$ \\
\hline 100 & $27 \cdot 078$ & $30 \cdot 147$ & $283 \cdot 49$ & 2270 & 0354 & $3 \cdot 267$ & 1.810 \\
\hline 150 & 58.35 & & & & & & \\
\hline 200 & $106 \cdot 43$ & & & & & & \\
\hline 250 & $170 \cdot 36$ & & & & & & \\
\hline
\end{tabular}

It will be noticed that although $\mathrm{X}$ and $\mathrm{Y}$ are derived in different ways, by taking certain values for $m$ and $n$ they could be made much the same in value, and altering $s$ or $l$ seems to produce the same sort of effects on $\mathrm{X}$ and $\mathrm{Y}$.

Mr. Beeston has drawn curves from the calculated numbers, but these need not be published.

If there is no self-induction, increasing the leakage increases the distance to which we can telephone. If there is no leakage, increasing the self-induction increases the distance.

When the amounts of $s$ and $l$ are not too great, increasing either increases the distance. These and other important facts are visible in the tables.

Without such tables as these and this method of study it would be almost impossible for the average mathematician to make anything of his mathematical results. Thus, for example, when equation (1) applies to such a function as $\sin p t$ it is just the same as

$$
\frac{d^{2} c}{d x^{2}}=\left(k l-\frac{s r}{p^{2}}\right) \frac{d^{2} c}{d t^{2}}+(k r+s l) \frac{d c}{d t} . \quad . \quad .
$$

Hence we see that the effect of leakage is to diminish the self-induction by the amount $s r / k p^{2}$, and to increase the 
resistance by the amount $s l / k$. But it is easy to see that if we diminish self-induction or increase resistance we do harm in telephony, and yet this kind of diminution through leakage does good. On going into the matter carefully, it is seen that it is the $p$ being in the denominator of $s r / k p^{2}$ which produces the good effect. In fact, if $l$ and $s$ are small, taking $p=6000, p^{\prime}=600$, we find

$$
\mathrm{X} \propto 1 / \sqrt{k r}\left\{1-4246 \frac{l}{r}-\frac{s}{3800 k}\right\} .
$$

So that increasing $s$ or $l$ produces a good effect. Having found the mathematical reason we have not far to go to find the physical reason.

It is evident from the tables that if we had no leakage we could completely get rid of the evil effects of capacity by introducing self-induction. It is also evident that if we had no self-induction, we could completely get rid of the evil effects of capacity by introducing leakage. But when there is some leakage and some self-induction, we can in practice only mitigate the evil effect of capacity ; for it is obvious that although certain values of $l$ and $s$ give infinite distances, doubling or halving these values produces enormous diminution in distance, and such a constant of a cable as $s$ may alter very greatly.

About fifteen years ago, with Prof. Ayrton I made many experiments on signalling through bare copper wires lying at the bottom of the water in the moat of Yedo in Japan. Here $k$ and $s$ were both very great. We had much less success than we expected, and we abandoned, perhaps too readily, our idea of a very cheap submarine cable.

The following tables are of general application. Let the numbers given in Table III. be divided by the value of $\sqrt{k} \overline{k r}$ for any cable or conductor of a telephonic line, and let them also be divided by the value of $m$ which is considered suitable*, and they will become the limiting distances $X$ in centimetres for that conductor, for the various values of $\frac{l}{r}$ and $\frac{s}{k}$ given.

Let the numbers in Table IV. be divided by the value of $\sqrt{k r}$ for any conductor of a telephonic line, and let them also be divided by the value of $n$ which is considered suitable, and they will become the limiting distances $\mathbf{Y}$ in centimetres for that conductor, for the various values of $\frac{l}{r}$ and $\frac{s}{k}$ given.

* It is more correct to say that the numbers are to be multiplied by $\log _{e}\left(1+\frac{1}{m}\right)$. 
Table III.

\begin{tabular}{|c|c|c|c|c|c|c|c|}
\hline \multirow[b]{2}{*}{$\begin{array}{l}\text { Values } \\
\text { of } s / k \text {. }\end{array}$} & \multicolumn{7}{|c|}{ Values of $10^{5} \times l / r$} \\
\hline & $\mathbf{0}$ & $1 \cdot 667$ & $16 \cdot 67$ & 50 & $83 \cdot 33$ & $116 \cdot 7$ & $166 \cdot 7$ \\
\hline 0 & $0 \cdot 0267$ & $0 \cdot 0286$ & 0.0533 & $0 \cdot 1404$ & $0 \cdot 2765$ & $0 \cdot 4753$ & $0 \cdot 9191$ \\
\hline 2154 & 0.0285 & 0.0307 & 0.0594 & $0 \cdot 1722$ & $0 \cdot 3787$ & $1 \cdot 1281$ & $2 * 053$ \\
\hline 600 & ...... & ...... & ...... & ....... & $\ldots \ldots$ & $\ldots \ldots$ & $\infty$ \\
\hline $430 \cdot 8$ & 0.0307 & 0.0332 & 0.0640 & $0 \cdot 2298$ & 0.5970 & $1 \cdot 6034$ & $8 \cdot 913$ \\
\hline 857 & ....... & ...... & ...... & $\ldots \ldots$ & ...... & $\infty$ & \\
\hline $861 \cdot 5$ & $0 \cdot 0358$ & 0.0392 & 0.0912 & $0 \cdot 4891$ & $3 \cdot 4732$ & 27550 & $3 \cdot 576$ \\
\hline 1200 & ...... & ...... & ...... & $\ldots \ldots$ & $\infty$ & & \\
\hline 1723 & 0.0476 & 0.0534 & $0 \cdot 1698$ & $10 \cdot 596$ & $1 \cdot 8455$ & $0 \cdot 5145$ & $0 \cdot 2377$ \\
\hline 2000 & $\ldots \ldots$ & $\ldots \ldots$ & $\ldots \ldots$ & $\infty$ & & & \\
\hline 3015 & 0.0686 & 0.0797 & $0-4799$ & 1.0792 & $0 \cdot 2099$ & $0 \cdot 1130$ & 0.0699 \\
\hline 4308 & $0 \cdot 0936$ & $0 \cdot 1123$ & $1 \cdot 9564$ & $0 \cdot 2717$ & $0 \cdot 0176$ & 0.0573 & 0.0439 \\
\hline 6000 & ...... & $\ldots \ldots$ & $\infty$ & & & & \\
\hline 60000 & ...... & $\infty$ & & & & & \\
\hline
\end{tabular}

Table IV.

\begin{tabular}{|c|c|c|c|c|c|c|c|}
\hline \multirow[b]{2}{*}{$\begin{array}{l}\text { Values } \\
\text { of } s / k \text {. }\end{array}$} & \multicolumn{7}{|c|}{ Values of $10^{5} \times l / r$} \\
\hline & 0 & $1 \cdot 667$ & $16^{\prime} 67$ & 50 & $83 \cdot 33$ & 116.7 & 1667 \\
\hline 0 & 0.0531 & 0.0540 & 0.0646 & 0.0969 & $0 \cdot 1352$ & $0 \cdot 1813$ & $0 \cdot 2633$ \\
\hline $215^{*} 4$ & 0.0687 & 0.0700 & $0 \cdot 0881$ & $0 \cdot 1506$ & $0 \cdot 2427$ & $0 \cdot 3808$ & 0.7379 \\
\hline 600 & $\ldots \ldots$ & $\ldots \ldots$ & $\ldots \ldots$ & $\ldots \ldots$ & $\ldots \ldots$ & $\ldots \ldots$ & $\infty$ \\
\hline $430 \cdot 8$ & 0.0882 & 0.0903 & 0.1199 & $0 \cdot 2414$ & 0.5139 & $1 \cdot 0502$ & $4 \cdot 231$ \\
\hline 857 & $\cdots+\cdots$ & ....... & $\ldots \ldots$ & $\ldots \ldots$ & $\ldots \ldots$ & $\infty$ & \\
\hline $861 \cdot 5$ & $0 \cdot 1364$ & $0 \cdot 1409$ & 0.2108 & 06800 & $3 \cdot 0033$ & 111056 & 2732 \\
\hline 1200 & ...... & ...... & $\ldots \ldots$ & $\ldots \ldots$ & $\infty$ & & \\
\hline 1723 & 02652 & $0 \cdot 2781$ & 0.4377 & $20 \cdot 596$ & 27837 & 06613 & $0 \cdot 2624$ \\
\hline 2000 & ....... & ...... & $\ldots \ldots$ & $\infty$ & & & \\
\hline 3015 & 0.5533 & 0.6306 & $2 \cdot 1079$ & $2 \cdot 775$ & 0.4179 & $0 \cdot 1915$ & $0 \cdot 1024$ \\
\hline 4308 & 0.9847 & $1 \cdot 0963$ & $0 \cdot 1031$ & 0.826 & 0.0129 & $0 \cdot 1188$ & $0 \cdot 0658$ \\
\hline 6000 & $\ldots \ldots$ & $\ldots \ldots$ & $\infty$ & & & & \\
\hline 60000 & $\ldots \ldots$ & $\infty$ & & & & & \\
\hline
\end{tabular}


Another way of putting the results in these tables is this :-Let $K$ be the whole capacity of the line in farads, $R$ its resistance in ohms, $L$ its whole self-induction in secohms, $S$ the whole leakage-conductivity in mhos : L/R and $\mathrm{S} / \mathrm{K}$ are the same as the $l / r$ and $s / k$ of the tables. For given values of these find the number in either table; square it and divide by any chosen $m^{2}$ or $n^{2}$; this gives the product of the whole capacity $K$ and the whole resistance $R$ of the conductor.

Of course in all lines which have the same values of $L / R$ and $S / K$, the product $K R$ is constant. 1 must again draw attention to the fact that we have neglected the terminal conditions.

I have not hitherto said anything about the amplitude of the current; in fact the receiving apparatus has been supposed to be infinitely delicate. . It is obvious that $1 / h$ is the distance in which the amplitude becomes $1 / \epsilon^{\text {th }}$ of its initial amount. If the numbers in the following Table V. be divided by $\sqrt{k r}$ for any line, they give the distances in which a current of frequency 955 per second has its amplitude halved.

\section{TABLE V.}

\begin{tabular}{|c|c|c|c|c|c|c|c|}
\hline \multirow{2}{*}{$\begin{array}{l}\text { Values } \\
\text { of } s / k \text {. }\end{array}$} & \multicolumn{7}{|c|}{ Values of $\frac{e}{-} \times 10^{5}$} \\
\hline & 0 & 1667 & 16.67 & 50 & $83 \cdot 3$ & 1167 & 1667 \\
\hline 0 & .0127 & .0167 & .0197 & .0314 & .0402 & .0476 & $\cdot 0567$ \\
\hline $215 * 4$ & $\cdot 0124$ & .0130 & .0188 & .0283 & .0340 & .0389 & .0331 \\
\hline $430 \cdot 8$ & .0122 & .0128 & .0177 & $\cdot 0257$ & $\cdot 0295$ & 0315 & .0329 \\
\hline $861 \cdot 5$ & .0118 & .0123 & .0167 & .0218 & .0233 & $\cdot 0236$ & .0232 \\
\hline 1723 & .0110 & .0114 & .0145 & .0166 & .0164 & .0158 & .0147 \\
\hline 3015 & .0099 & $\cdot 0102$ & $\cdot 0121$ & .0124 & $\cdot 0115$ & .0106 & 0096 \\
\hline 4308 & .0091 & .0093 & .0105 & .0100 & .0090 & .0081 & .0072 \\
\hline 6462 & .0079 & & & & & & \\
\hline 8616 & .0071 & & & & & & \\
\hline 10770 & .0065 & & & & & & \\
\hline
\end{tabular}

The effect of leakage is in every case to diminish the amplitude of the current, making it necessary to have more sensitive receiving-instruments or more powerful sending-instruments.

If some of our clever mathematicians would for a while put aside the ambition to write original papers and would 
give us in one paper, however long, an exposition of $\mathrm{Mr}$. Heaviside's views on this subject, he would confer great benefits upon the average electrician. Mr. Heaviside can discover new truths, and we all believe in his results when we understand them, but he seems unable to lower his reasoning to our mathematical levels. Since writing this paper I have tried to understand Mr. Heaviside's numerous papers on this subject, but I am sorry to say that I am not yet able to express a certain opinion as to the practical value, or want of value, of the preceding tables.

\section{Proceedings of Learned Societies.}

\section{GEOLOGICAL SOCIETY.}

[Continued from p. 150.]

$$
\begin{aligned}
& \text { June 7th, 1893.-W. H. Hudleston, Esq., M.A., F.R.S., } \\
& \text { President, in the Chair. }
\end{aligned}
$$

THE following communications were read :-

1. "The Bajocian of the Sherborne District: its Relations to Subjacent and Superjacent Deposits." By S. S. Buckman, Esq., F.G.S.

This paper is partly the result of excavations made by Mr. Hudleston, F.R.S., and the author at Sherborne, to determine the position of the so-called 'Sowerbyi-zone.'

The author uses the term 'Bajocian' in a merely conventional sense to denote the lower beds of what has been called "Upper part of the Inferior Oolite.' $\mathrm{He}$ introduces a term emar ( $\boldsymbol{\eta}_{\mu a \rho}$ ) as a chronological subdivision of an 'age,' and considers the beds dealt with in the paper to have been deposited during 12 emata, which he calls, in descending order, fuscum, zigzag, Truellii, Garantianum, niortense, Humphriesianum, Sauzei, Witchellia sp., discites, concavum, bradfordense, and Murchisonce.

A line from Stoford, Somerset, through North Dorset to Milborne Wick, Somerset, is the base-line of the district reviewed. Seventeen sections of places close to this line are given to show the relations of the beds, with the different amounts of strata deposited during successive emata, and during the same emar at different places. By means of Tables he shows that the area of maximum accumulation receded eastwards in the earlier emata, and then proceeded westwards during the later emata. A similar and corresponding faunal recession and progression is pointed out, though the faunal headquarters always remain west of the great accumulation of deposit. Adding the various maximum deposits together, the author finds as much as 130 feet of strata deposited during the twelve emata, = (practically) the 'Inferior Oolite of Dorset.' This is a far greater thickness than has hitherto been allowed to beds of 